\section{Benchmarks for outcome indicators in pediatric cataract surgery}

\begin{abstract}
Purpose The purpose of this study was to establish benchmarks for outcome indicators that may help ascertain the quality of pediatric cataract surgery with primary intraocular lens (IOL) implantation.

Patients and methods A retrospective chart review of patients older than 2 years undergoing cataract surgery with primary IOL implantation, by multiple surgeons in a tertiary-care center, from November 2005 to February 2016 was conducted. Patients with ocular comorbidities that would affect the outcomes were excluded. The outcome measures chosen were as follows: (1) final best corrected Snellen visual acuity (BCVA) in patients who had bilateral cataract surgery analyzed at the last clinic visit; (2) prediction error $(\mathrm{PE})=$ expected refraction - actual refraction. Mean PE and mean absolute PE were assessed 1 month postoperatively, irrespective of age or laterality.

Results Mean age at surgery was $8.3 \pm 4.6$ years and mean follow-up duration was $3.7 \pm 2.7$ years. The results of outcome measures were as follows: (1) BCVA was $20 / 40$ or better in $96 \%(n=124$ eyes, mean patient age: $8.3 \pm 4.6$ years). Remaining five eyes had amblyopia with two eyes having BCVA worse than 20/100 that did not respond to amblyopia treatment. (2) Mean PE was $0.3 \pm 1.1 \mathrm{D}$ and mean absolute $\mathrm{PE}$ was $0.9 \pm 0.7 \mathrm{D}$. PE was within $\pm 0.5 \mathrm{D}$ in $43.0 \%$, $\pm 1.0 \mathrm{D}$ in $66 \%$, and $\pm 2.0 \mathrm{D}$ in $95 \%$ ( $n=235$ eyes).

Conclusion Good visual acuity after cataract surgery should be expected for children with bilateral cataracts, setting a high benchmark similar to that recommended in adult cataract surgery. Prediction error is greater in pediatric eyes than in adult eyes, setting a lower benchmark. This study establishes benchmark for outcome indicators in
\end{abstract}

BR Nihalani and DK VanderVeen

pediatric patients older than 2 years undergoing cataract surgery with primary IOL implantation.

Eye (2017) 31, 417-421; doi:10.1038/eye.2016.240;

published online 4 November 2016

\section{Introduction}

Quality assurance programs have been instituted in many hospitals to improve outcomes. In the United States, governmental initiatives to encourage outcomes reporting were first introduced in 2008 as the Physician Quality Reporting Initiative (PQRI), now known as Physician Quality Reporting System (PQRS). The primary purpose of these quality assurance programs was to identify the potential for improving outcomes, which requires identification of and reporting measures that are meant to reflect quality of care. It is important to adopt indicators that unify and simplify often complex medical interventions to create a common base for comparing the performance and the outcome of different providers. ${ }^{1}$

The most commonly used indicators in adult cataract surgery are best corrected visual acuity (BCVA) and refractive accuracy as reported in multiple benchmark studies. ${ }^{1-7}$ However, there are no publications focused on benchmark indicators in pediatric cataract surgery, probably because pediatric patient populations usually include 'complicated' cases, with unique surgical and management challenges, including inability to obtain accurate biometry or cooperate for reliable visual acuity (VA) testing. Young children have smaller eyes with shorter globe axial length (AL), and potentially anterior segment anatomic abnormalities that can affect effective biometry measures, intraocular lens (IOL) calculations, and effective IOL position and thus post-operative refraction. Moreover, young children are often intentionally left with variable degrees of hyperopic refractive error
Department of Ophthalmology, Children's Hospital Boston, Harvard Medical School, Boston, MA, USA

Correspondence: BR Nihalani, Department of Ophthalmology, Children's Hospital Boston, Harvard Medical School, 300 Longwood Avenue, Fegan 4, Boston, MA 02115, USA Tel: +1 6173556401 ; Fax: +1 6177300392 . E-mail: bharti.gangwani@ childrens.harvard.edu

Received: 5 April 2016 Accepted in revised form: 15 September 2016 Published online: 4 November 2016 
depending on the age at surgery, to allow for ocular growth after surgery and in anticipation of myopic shift. All of these differences make it difficult to assess and compare the outcomes of pediatric cataract surgery to adult outcomes.

The aim of our study was to establish valid benchmark indicators in the pediatric population, when uncomplicated surgery with primary IOL implantation is planned.

\section{Materials and methods}

The data included in this study were collected as part of the hospital quality assurance program, hence informed consent was not required from the patients. Approval from the institutional review board was obtained for this chart review.

This was a retrospective chart review of all patients older than 2 years of age undergoing cataract surgery with primary IOL implantation over an 11-year period (November 2005-February 2016). The patients with ocular comorbidities known to potentially result in poor vision or inability to obtain accurate biometry (corneal opacity, glaucoma, uveitis, trauma, manifest nystagmus, retinal disease, or subluxated lenses) were excluded from the study. The data collected included pre- and postoperative VA measures, biometry data, and refractive outcomes. The pre- and post-operative VA was measured with M\&S system (M\&S Technologies Inc., Niles, IL, USA). Post-operative retinoscopy and manifest refraction was performed within 90 days of surgery. Post-operative refraction and glasses prescription management was performed by the surgeon.

The method of pre-operative biometry depended on the age and cooperation of the child, as well as the density of the cataract. Optical biometry (Zeiss IOLMaster, Zeiss Humphrey Systems, Dublin, CA, USA) was attempted in most patients who were older and cooperative.

Keratometry and ultrasound biometry measures were otherwise taken by the surgeon. Keratometry was measured with a hand-held keratometer (Nidek Inc., Fremont, CA, USA), either in the office or under anesthesia. Contact ultrasound biometry (Sonomed E-Z scan AB 5500, Sonomed Inc., Lake Success, NY, USA) was performed in cooperative patients in the office, and for younger or uncooperative patients, under anesthesia. Immersion ultrasound was performed for most patients who had biometry performed under anesthesia. Ultrasound biometry was often repeated under anesthesia to confirm accuracy of measurements obtained in the office in younger, less cooperative children. The IOL calculation formula, IOL type, and refractive target were chosen by the surgeon depending on the age at surgery. SRK/T formula was used for IOL calculation in most eyes and single piece acrylic IOL was used in all these eyes. The surgical technique also varied somewhat depending on the operating surgeon; there were eight different surgeons that performed the surgeries in this study. All children from 2 to 5 years of age received posterior capsulotomy and anterior vitrectomy, and children older than 5 years did not receive posterior capsulotomy or anterior vitrectomy. The second eye of patients with bilateral cataracts was operated depending on the density and progress of cataract in that eye.

To assess cataract surgery performance, we studied the following two separate outcome measures:

\section{Best corrected visual acuity}

To evaluate VA in a consistent manner, we included only patients aged 2 years or older at the time of surgery, who were treated with bilateral cataract surgery, who were followed for at least 3 months after surgery, and who had a final VA measured with Snellen letters. In this manner, we eliminated the possibility of dense amblyopia from congenital cataract with late presentation, or those with dense amblyopia due to unilaterality. BCVA is the preferred visual outcome measure, as young children are often left intentionally with varying degrees of hyperopic refraction, due to expected ocular growth and myopic shift over time, and decreased uncorrected VA would not represent a poor outcome.

\section{Prediction error}

Prediction error (PE) and absolute PE was calculated in the following manner:

$\mathrm{PE}=$ predicted refraction - actual refraction

Absolute $\mathrm{PE}=$ |predicted refraction - actual refraction $\mid$

Predicted refraction was calculated for the IOL power that was implanted in the eye using $\mathrm{AL}, \mathrm{K}$, and the manufacturer's A-constant. The actual refraction was converted into spherical equivalent by using the formula:

Spherical equivalent $=$ Sphere + Cylinder $/ 2$.

The PE outcome was determined in patients irrespective of the age at surgery and laterality, thus it also included patients with unilateral cataracts and patients who were still too young for Snellen VA measurements.

We also sought to study differences in PE and refractive outcome based on short AL and age. Unpaired $t$-test was used to compare the mean PE and mean absolute PE between the two groups of patients depending on $\mathrm{AL}$ (AL $\leq 22 \mathrm{~mm}$ vs $\mathrm{AL}>22 \mathrm{~mm}$ ). One-way analysis of variance test was used to compare the PE in groups of patients depending on the age at surgery. $P$-value $<0.05$ was considered significant. We tabulated the number of 
patients who achieved a post-operative refraction within $0.5,1.0$, and 2.0 diopters (D) of the targeted refraction.

\section{Results}

One hundred and twenty-four eyes of 62 patients with bilateral cataract surgery met the inclusion criteria and were analyzed for VA outcomes. Mean age at surgery in this group was $8.3 \pm 4.6$ years (median age: 6.8 years).

Mean follow-up duration was $3.7 \pm 2.7$ years. All but one patient had IOL implantation within the capsular bag. The IOL power was reduced by $1.0 \mathrm{D}$ in the patient with sulcus implantation.

BCVA at the last visit was 20/40 or better in $96 \%$ eyes (Table 1) at their last follow-up visit (median follow-up; 3.0 years). When the eyes were classified depending on the age at surgery, there was no difference in median VA between different age groups as shown in Table 2. Five patients had amblyopia, of these three patients were in the age range of 2-6 years and one patient was in the age range of $6-10$ years, and one patient was $>10$ years at the time of cataract surgery. Two eyes had vision worse than 20/100 at the last visit. Both these patients were 3.7 and 4.5 years at the time of surgery and had severe deprivation amblyopia (BCVA: 20/100 and 20/200) that did not respond to amblyopia treatment. The VA had improved in all other patients compared with the preoperative measure.

PE calculations could be performed for 235 eyes of 179 patients (56 bilateral, 123 unilateral). The mean age at surgery in this group was $6.3 \pm 4.8$ years (median, 4.7 years; range, $0.1-18$ years). The mean $\mathrm{AL}$ was $22.3 \pm 1.8 \mathrm{~mm}$ (range, 17.7-27.8 mm). The mean PE was $0.3 \pm 1.1 \mathrm{D}$ (median, $0.375 \mathrm{D}$ ) and the mean absolute PE was $0.9 \pm 0.7 \mathrm{D}$ (median, $0.7 \mathrm{D}$ ). The PE was within $0.5 \mathrm{D}$ in $43 \%$ eyes, $1.0 \mathrm{D}$ of target in $66 \%$ eyes, and $2.0 \mathrm{D}$ of target in $95 \%$ eyes.

Table 3 shows the mean PE and mean absolute PE when the eyes were analyzed after classifying depending on the AL (eyes with $\mathrm{AL} \leq 22 \mathrm{~mm}$ vs $\mathrm{AL}>22 \mathrm{~mm}$ ). The mean absolute PE in the group of eyes with shorter AL was higher compared to eyes with long AL and the difference was significant statistically.

Table 4 compares the mean PE in four different age groups: age $\leq 1$ year $(n=19)$, age $>1-3$ years $(n=55)$, age
$>3-6$ years $(n=70)$, and age $>6-18$ years $(n=95)$. The mean absolute PE was not statistically significant between different age groups.

\section{Discussion}

Quality management based on outcome indicators is increasingly finding its way into Ophthalmology practices, especially for adult cataract and refractive surgery. ${ }^{1-7}$ To evaluate performance, a reference for good quality or best practice is needed, the so-called benchmark. It is increasingly important to establish benchmarks in the face of initiatives by the government, hospitals, and insurance companies to improve quality of care and effectiveness. ${ }^{1}$

Pediatric patients often represent 'complicated' cases as the surgery can be technically challenging compared with the adult cataract surgery owing to highly elastic lens capsule, low scleral rigidity, small compliant eyes, and the need for primary management of posterior capsule and anterior vitreous due to higher incidence of visual axis opacification. In addition, despite the best technical outcomes, functional vision outcomes can be less reliable not only due to variable cooperation for vision testing, but VA measures can be compromised by co-existing conditions such as amblyopia, latent nystagmus, strabismus, or intentionally planned residual refractive error, which must be managed concurrently in a young child. We measured final BCVA in our cohort to allow for recovery of vision for patients in the amblyogenic age group. Unilateral cataract patients were excluded, as amblyopia in these cases is often too dense to overcome even with good compliance to therapy, and final BCVA is not a reflection on cataract surgery quality of care. Pediatric patients are also often excluded from adult PE studies due to inconsistencies with biometry technique,

Table 1 Best corrected visual acuity (BCVA) outcomes in patients older than 2 years with bilateral cataract surgery

\begin{tabular}{lrc}
\hline BCVA & N & Percentage of eyes \\
\hline $20 / 20-20 / 30$ & 106 & $85 \%$ \\
$>20 / 30-20 / 40$ & 13 & $11 \%$ \\
$<20 / 40$ & 5 & $4 \%$ \\
\hline
\end{tabular}

$n=124$ eyes.

Table 2 Median visual acuity in eyes operated for bilateral cataract surgery classified depending on age at surgery

\begin{tabular}{lcccc}
\hline Age at surgery & Median age at surgery & $\mathrm{N}$ & Median BCVA & No. of amblyopic eyes \\
\hline 2-6 years & 4.3 years & 43 & $20 \times 25$ & 3 \\
$>6-10$ years & 7.8 years & 28 & $20 \times 25$ & 1 \\
$>10-18$ years & 14.2 years & 54 & $20 \times 25$ & 1 \\
\hline
\end{tabular}

Abbreviation: BCVA, best corrected visual acuity. 
Table 3 Prediction error (PE) by axial length (AL)

\begin{tabular}{lccc}
\hline$A L$ & $\mathrm{~N}$ & Mean $P E^{\mathrm{a}}$ & Mean absolute $P E^{\mathrm{b}}$ \\
\hline$\leq 22 \mathrm{~mm}$ & 103 eyes & $0.4 \pm 1.3 \mathrm{D}$ & $1.0 \pm 0.8 \mathrm{D}$ \\
$>22 \mathrm{~mm}$ & 132 eyes & $0.2 \pm 0.9 \mathrm{D}$ & $0.8 \pm 0.6 \mathrm{D}$ \\
\hline
\end{tabular}

$n=235$ eyes. ${ }^{a} P=0.2$, unpaired $t$-test, not significant for mean PE. ${ }^{\mathrm{b}} P=0.02$, unpaired $t$-test, statistically significant for mean absolute PE.

Table 4 Prediction error (PE) by age

\begin{tabular}{llrc}
\hline Age & $\mathrm{N}$ & Mean $P E$ & Mean absolute $P E$ \\
\hline$\leq$ 1 year & 19 & $-0.1 \pm 1.5$ & $0.8 \pm 0.9$ \\
$>$ 1-3 years & 55 & $0.1 \pm 1.2$ & $0.9 \pm 0.8$ \\
$>$ 3-6 years & 70 & $0.5 \pm 0.1$ & $0.9 \pm 0.7$ \\
6-18 years & 95 & $0.3 \pm 1.1$ & $0.9 \pm 0.7$ \\
\hline
\end{tabular}

$n=235$ eyes. $P=0.05$, one-way analysis of variance, significant for mean PE. $P=0.9$, one-way analysis of variance, not significant for mean absolute PE.

and because of inherent difficulties of obtaining accurate biometry in small compliant eyes. Optical biometry is often not possible either due to lack of cooperation or density of the cataract. Post-operative refractions can be challenging. There is no IOL calculation formula specifically designed for pediatric eyes, but surgeons generally use a preferred formula based on AL. Eyes with globe $\mathrm{AL}<22 \mathrm{~mm}$ are prone to greater $\mathrm{PE}$, and while uncommon in adult populations, are typical in children. Use of optimized constants is often not possible due to lack of consistent data from a large number of eyes having similar anatomic characteristics and ALs operated by a single surgeon. Furthermore, due to the continued growth of the eye and changing refractive status, a smaller window is needed to obtain the post-operative refraction and assess PE, and the final refractive status and vision may not be known for many years. PE was greater in our population compared with that reported in adult studies, ${ }^{1,4,6}$ likely due to measurement error in these small compliant eyes or lack of customized constants for IOL formula calculations.

The children with bilateral cataract surgery in our study achieved excellent VA outcomes, which is comparable to outcomes reported for surgery in adult eyes. Hahn et al ${ }^{1}$ conducted a prospective multicenter cohort study with seven participating centers, and analyzed visual outcomes in 1553 adult patients. They reported VA of 20/40 or better in $98.5 \%$ patients 3 months after surgery. Jaycock et $a l^{5}$ used the cataract national data set comprising 55567 adult cataract operations using multicenter data from an electronic patient record system to establish benchmark standards of care in the National Health Service (NHS) and beyond. Post-operative VA of $20 / 40$ or better was achieved in $91 \%$ of eyes; $94.7 \%$ in eyes with no copathologies, and $79.9 \%$ in eyes with one or more copathologies. Ledoux et $a l^{8}$ analyzed VA outcome in patients with pediatric cataract extraction with IOL implantation older than 4 years at the time of surgery and found that $80.8 \%$ (59/73 patients) with bilateral cataract surgery had visual outcome of 20/40 and better. Crouch et $a l^{9}$ performed a prospective analysis of pediatric pseudophakia in children between 12 months and 18 years, and reported that $95 \%$ of patients with bilateral cataract surgery had VA of $20 / 30$ or better ( $n=20$ eyes of 10 patients), though the sample size in this study was small.

PE reflects the accuracy of a surgeon to obtain the expected post-operative refractive target, and is a good benchmark indicator of refractive outcomes in the immediate post-operative period. Another way to report refractive outcomes is by calculating the number of eyes within an acceptable range from the expected refractive target. In the Hahn et al ${ }^{1}$ multicenter study, surgeons achieved a spherical equivalent within $1.0 \mathrm{D}$ of the targeted refraction in $95.5 \%$ eyes, and within $0.5 \mathrm{D}$ in $77.4 \%$ of the cases, measured 1 month after surgery. Simon et $\mathrm{al}^{4}$ reported a target refraction within $1 \mathrm{D}$ in $94 \%$ of eyes $(n=1275)$ analyzed at an academic teaching institution's comprehensive ophthalmology service, measured 90 days after the surgery. Gale et $a l^{6}$ established benchmark standards for refractive outcome after adult cataract surgery in the NHS by analyzing three cycles of prospective data where the electronic medical record automatically recommended the IOL calculation formula using customized A constant. The post-operative subjective refraction was within $1 \mathrm{D}$ of the predicted value in $80 \%$ eyes in cycle $1,83 \%$ in cycle 2 , and $87 \%$ in cycle 3 . This report supports the idea that measurement of refractive outcomes can allow changes in practice and result in improvement in this quality of care measure.

The mean absolute PE was somewhat greater in our general pediatric population than reported in many adult populations, at $0.9 \pm 0.7 \mathrm{D}$, and we note that typical absolute PE is reported to be between $0.7 \mathrm{D}$ and $1.5 \mathrm{D}$ in pediatric populations. ${ }^{10-13}$ We previously reported a subset of these patients where the mean absolute PE was $0.97 \pm 0.91$ in children $\leq 2$ years $(N=22)$ and $0.85 \pm 0.69$ in children $>2$ years $(N=113)$, which was not statistically different though the sample size of children $<2$ years was very small. In the same population, the mean absolute PE was $1.08 \pm 0.77$ in children with $\mathrm{AL} \leq 22 \mathrm{~mm}(N=69)$, which was statistically different than mean absolute PE of $0.75 \pm 0.64$ in children with $\mathrm{AL}>22 \mathrm{~mm}(N=66) .{ }^{12}$ The Infant Aphakia Treatment Study reported mean absolute PE of $1.8 \pm 1.3 \mathrm{D}$ for infantile eyes with primary IOL surgery. ${ }^{14}$ In the IATS, refractions were within $0.5 \mathrm{D}$ in $12 \%$ eyes, within $1 \mathrm{D}$ in $45 \%$ of eyes, and within $2 \mathrm{D}$ in 
$59 \%$ of eyes, with an inverse relationship between PE and AL.

The limitations of our study are that it is retrospective, and it includes pediatric patients with a wide range of ages at surgery. On the other hand, this population is fairly representative of the typical pediatric cataract population in a tertiary referral center, noting our inclusion and exclusion criteria for the outcomes studied. A strength of the study is the large number of eyes, operated on by multiple surgeons with varying degrees of experience, though all surgeons performed current surgical techniques using modern foldable IOLs. With regard to visual outcomes, because children were selected by surgical technique (primary IOL implantation), bilateral cataract status, and ability to cooperate for Snellen VA testing, we may have induced a bias for better BCVA outcomes. In addition, we did not include children with co-existing ocular anatomic abnormalities or systemic diagnoses that might interfere with ability to obtain accurate VA testing, so VA scores may not be as good, or as easily measured, for such children. With regard to benchmarks for post-operative refraction, we find that more variability should be expected with veryshort eyes and in very-young children.

For quality outcome measures, we suggest that children $>2$ years at the time of bilateral cataract surgery with primary IOL implantation should achieve excellent visual outcomes in $96 \%$ of eyes, which is comparable to benchmarks in adult eyes. The limiting factor in pediatric eyes is amblyopia. The PE in pediatric eyes is somewhat greater than benchmark reported in adult population studies, so that overall only $66 \%$ are within $1 \mathrm{D}$ of the target refraction.

\section{Summary}

What was known before

- There are only a few published studies on outcomes of pediatric cataract surgery and these were not intended to establish benchmark indicators for outcomes in pediatric eyes.

\section{What this study adds}

- This study establishes benchmark indicators for outcomes of pediatric cataract surgery for visual acuity and refractive outcomes in children older than 2 years at the time of surgery.

\section{Conflict of interest}

The authors declare no conflict of interest.

\section{References}

1 Hahn U, Krummenauer F, Kolbl B, Neuhann T, SchayanAraghi K, Schmickler S et al. Determination of valid benchmarks for outcome indicators in cataract surgery. A multicenter, prospective cohort trial. Ophthalmology 2011; 118: 2105-2112.

2 Hahn U, Krummenauer F, Neuhann I. Result related success rates of cataract operations. Results of a systemic literature review. Ophthalmology 2012; 109: 575-582.

3 Zaidi FH, Corbett MC, Burton BJL, Bloom PA. Raising the benchmark for the 21st century-the 1000 cataract operations audit and survey: outcomes, consultantsupervised training and sourcing NHS choice. $\mathrm{Br} \mathrm{J}$ Ophthalmol 2007; 91: 731-736.

4 Simon SS, Chee YE, Haddadin RI, Veldman PB, Borboli-Gerogiannis S, Brauner SC et al. Achieving target refraction after cataract surgery. Ophthalmology 2014; 121: 440-444.

5 Jaycock P, Johnston RL, Taylor H, Adams M, Tole DM, Galloway $\mathrm{P}$ et al. The cataract national dataset electronic multicenter audit of 55567 operations: updating benchmark standards of care in the United Kingdom and internationally. Eye 2009; 23: 38-49.

6 Gale RP, Saldana M, Johnston RL. Benchmark standards for refractive outcomes after NHS refractive surgery. Eye 2009; 23: 149-152.

7 Murphy C, Tuft SJ, Minassian DC. Refractive error and visual outcome after cataract extraction. J Cataract Refract Surg 2002; 28: 62-66.

8 Ledoux DM, Trivedi RH, Wilson Jr ME, Payne JF. Pediatric cataract extraction with intraocular lens implantation. Visual acuity outcome when measured at age 4 years and older. J AAPOS 2007; 11: 218-224.

9 Crouch ER, Crouch Jr ER, Pressman SH. Prospective analysis of pediatric pseudophakia: myopic shift and postoperative outcomes. J AAPOS 2002; 6: 277-282.

10 Andreo LK, Wilson ME, Saunders RA. Predictive value of regression and theoretical IOL formulas in pediatric intraocular lens implantation. J Pediatr Ophthalmol Strabismus 1997; 34(4): 240-243.

11 Neely DE, Plager DA, Borger SM, Golub RL. Accuracy of intraocular lens calculations in infants and children undergoing cataract surgery. J AAPOS 2005; 9(2): 160-165.

12 Nihalani BR, VanderVeen DK. Comparison of intraocular lens power calculation formulae in pediatric eyes. Ophthalmology 2010; 117(8): 1493-1499.

13 Trivedi RH, Wilson ME. Prediction error after pediatric cataract surgery with intraocular lens implantation: contact versus immersion A-scan biometry. J Cataract Refract Surg 2011; 37(3): 501-505.

14 VanderVeen DK, Trivedi RH, Nizam A, Lynn MJ, Lambert SRInfant Aphakia Treatment Study Group. Predictability of intraocular lens power calculation formulae in infantile eyes with unilateral congenital cataract: results from the Infant Aphakia Treatment Study. Am J Ophthalmol 2013; 156: 1252-1260. 